\title{
Multiple reflection expansion and heat kernel coefficients
}

\author{
M. Bordag* and D. Vassilevich ${ }^{\dagger}$ \\ University of Leipzig, Institute for Theoretical Physics, Augustusplatz 10/11, 04109 Leipzig, Germany \\ H. Falomir ${ }^{\ddagger}$ and E. M. Santangelo ${ }^{\S}$ \\ Departamento de Física, Universidad Nacional de La Plata, C.C.67, 1900 La Plata, Argentina
}

(Received 30 March 2001; published 30 July 2001)

\begin{abstract}
We propose the multiple reflection expansion as a tool for the calculation of heat kernel coefficients. As an example, we give the coefficients for a sphere as a finite sum over reflections, obtaining as a byproduct, a relation between the coefficients for Dirichlet and Neumann boundary conditions. Further, we calculate the heat kernel coefficients for the most general matching conditions on the surface of a sphere, including those cases corresponding to the presence of delta and delta prime background potentials. In the latter case, the multiple reflection expansion is shown to be nonconvergent.
\end{abstract}

DOI: $10.1103 /$ PhysRevD.64.045017

PACS number(s): 12.20.Ds, 03.70.+k, 42.50.Lc

\section{INTRODUCTION}

Heat kernel coefficients play an important role in many areas of theoretical physics. They govern the short-distance behavior of the propagator and the small-time asymptotics of the Schrödinger equation. In quantum-field theory, heat kernel coefficients define the one-loop counterterms and quantum anomalies, as well as the large mass expansion of the effective action [1]. It is clear, therefore, that it is important to have an effective method of calculation of these coefficients.

To the best of our knowledge, heat kernel methods were first applied to quantum physics by Fock in 1937 [2]; then, they were reintroduced by Schwinger in the 1950s (see Ref. [3]). Because of DeWitt [4], these methods became standard in quantum-field theory. The DeWitt iteration procedure proved to work quite well on manifolds without boundaries and (after certain improvements) allowed for the calculation of many terms in the asymptotic expansion of the heat kernel [5-8]. On manifolds with boundaries, the methods based on functorial properties of the heat kernel [9-11] appeared to be more appropriate. These methods allowed for the calculation of some higher terms of the heat kernel expansion, e.g., for local boundary conditions [12] and for a transmittal problem [13]. Even though the functorial methods are the most general and the most powerful ones, they still have their limitations. They work particularly well for most general operators in a certain category. However, the number of independent invariants, which can enter a heat kernel coefficient, grows very fast with the order of the asymptotic expansion, so that combinatorics becomes unmanageable. Alternatively, the general Seeley calculus, which is applicable for general boundaries, may be used. But this method becomes unwieldy beyond low orders.

The methods mentioned above are analytical, i.e., they

\footnotetext{
*Email address: Michael.Bordag@itp.uni-leipzig.de

†Email address: vassil@itp.uni-leipzig.de

\$Email address: falomir@obelix.fisica.unlp.edu.ar

§mail address: mariel@obelix.fisica.unlp.edu.ar
}

produce local formulas for the heat kernel coefficients in terms of the relevant geometric invariants. An alternative to such methods are special case calculations (see, e.g., Refs. [14-18] and references therein). In this case, the complexity of the calculations is almost independent from the order of the asymptotic expansion, but the method can only be applied to those problems where a sufficiently high symmetry allows for the separation of variables. Some recent examples of how the special case calculations may be combined with the analytical methods, can be found in Refs. [19,20,12,13].

Another alternative is provided by iterative, respectively, recursive methods. Well known is the DeWitt iteration method. Less known are reformulations in terms of integral equations. For example, in Ref. [21], the LipmannSchwinger equation for the scattering problem was used to determine the asymptotic expansion of the Jost function entering the regularized ground-state energy. While the known iterative methods work well for sufficiently smooth background fields, an effective method working for singular background fields or for boundary conditions is missing.

The aim of the present paper is to suggest the multiple reflection expansion as such a method. In fact, it is based on an integral equation whose kernel is located on the boundary. The iteration of this equation gives rise to the multiple reflection expansion. The important point is that only a finite number of reflections contribute to a given heat kernel coefficient.

The use of the multiple reflection expansion, in connection with vacuum energy, is not new. In Ref. [22] it was employed to investigate the asymptotic density of eigenvalues which, in the modern language, is equivalent to the calculation of heat kernel coefficients. In Ref. [23], the possibility of using the multiple reflection expansion was mentioned, but found to be too complicated for a general boundary. As far as we know, the method has never been used as a tool for the calculation of the heat kernel coefficients. However, it should be noticed that the corresponding integral equations (with kernel on the boundary surface, see Sec. II) have been used for proving existence and uniqueness theorems of Dirichlet and Neumann problems; see, for example, Ref. [24]. In this paper, we demonstrate its effective- 
ness with a recalculation of the heat kernel coefficients on a sphere. As a nice byproduct, we obtain a representation of the coefficients as finite sums, where the difference between Dirichlet and Neumann boundary conditions resides in certain signs only.

In general, the multiple reflection expansion can be viewed as some kind of perturbative expansion. For instance, for imaginary frequencies, it provides a well convergent series for the propagator. It should be mentioned that, in certain cases (for instance, with Dirichlet boundary conditions), this is so despite the absence of a small expansion parameter. So, the question is whether this convergent behavior is a general feature. The answer is no, and we provide a counterexample by considering the most general matching conditions on the surface of a sphere. They are described by a four-parameter family and correspond, for instance, to the presence of a delta function or its derivative on the surface. It turns out that there is no expansion in powers of the parameter in front of the derivative of the delta function but, instead, a nice expansion in the inverse of this parameter, which cannot be obtained at all by a multiple reflection expansion. We would like to note that a Green's function with a $\delta^{\prime}$-function potential has been considered before, for example, by path-integral methods in Ref. [25]. There, it was noticed that a perturbative expansion similar to that for a $\delta$-function potential yields some unsolvable relations, a fact that is not surprising in view of the nonanalyticity found by us.

The paper is organized as follows. In Sec. II we collect the necessary formulas on spectral functions and their relations to the heat kernel coefficients. We write down the multiple reflection expansion in terms of integral equations for the propagator as well as for the heat kernel. In Sec. III we use the multiple reflection expansion in order to reobtain the heat kernel coefficient for the classical boundary conditions on the sphere. In Sec. IV, we consider the most general matching conditions on a sphere, and calculate the corresponding heat kernel coefficients. Section V contains a discussion of our results and the conclusions. Some useful formulas are banned into Appendix A, while Appendix B contains the study of matching conditions in higherdimensional spaces.

\section{SPECTRAL FUNCTIONS}

In this section, we define the spectral functions to be used in the rest of the present paper, and give a short introduction to perturbative methods, supplemented with some examples of their application.

Let us consider the Laplace operator on a domain $\Omega$ $\in \mathbb{R}^{D}$. Let $\Phi_{n}(\vec{x})$ be its eigenfunctions, fulfilling Dirichlet or Neumann (or, more generally, Robin) boundary conditions on $S=\partial \Omega, \lambda_{n}$ being the corresponding eigenvalues

$$
-\Delta \Phi_{n}(\vec{x})=\lambda_{n} \Phi_{n}(\vec{x})
$$

(In Sec. IV we will consider the more complicated case of matching conditions on a surface in $\mathbb{R}^{D}$.) We consider three local spectral functions. The first one is the resolvent kernel (propagator) $D_{\omega}(\vec{x}, \vec{y})$ (at imaginary frequency), obeying the equation

$$
\left(\omega^{2}-\Delta\right) D_{\omega}(\vec{x}, \vec{y})=\delta(\vec{x}-\vec{y}) .
$$

It can be represented as

$$
D_{\omega}(\vec{x}, \vec{y})=\sum_{n} \frac{\Phi_{n}(\vec{x}) \Phi_{n}(\vec{y})}{\omega^{2}+\lambda_{n}} .
$$

The second spectral function is the zeta function, given by

$$
\zeta(\vec{x}, \vec{y} ; s)=\sum_{n} \lambda_{n}^{-s} \Phi_{n}(\vec{x}) \Phi_{n}(\vec{y})
$$

and, finally, the third is the heat kernel

$$
K(\vec{x}, \vec{y} \mid t)=\sum_{n} \Phi_{n}(\vec{x}) \Phi_{n}(\vec{y}) e^{-t \lambda_{n}} .
$$

These functions are related by means of

$$
\begin{aligned}
\Gamma(s) \zeta(\vec{x}, \vec{y} ; s) & =\int_{0}^{\infty} d t t^{s-1} K(\vec{x}, \vec{y} \mid t) \\
& =\frac{2}{\Gamma(1-s)} \int_{0}^{\infty} d \omega \omega^{1-2 s} D_{\omega}(\vec{x}, \vec{y}) .
\end{aligned}
$$

In addition, we consider the corresponding global quantities, which appear as integrals over $\Omega$ of the local ones in the coincidence limit. Because of the distributional character of the heat kernel coefficients $a_{n}(\vec{x}, \vec{x})$, it is useful to introduce a test function $f(\vec{x})$ into this integration. So let

$$
\begin{aligned}
\zeta[f](s) & =\int_{\Omega} d x f(x) \zeta(\vec{x}, \vec{x} ; s), \quad \text { respectively, } \\
K[f](t) & =\int_{\Omega} d x f(x) K(\vec{x}, \vec{x} \mid t)
\end{aligned}
$$

be the global zeta function (respectively, heat kernel). The fiber (matrix) trace has to be understood in the integrands. In many cases (as, e.g., for manifolds with boundaries and local boundary conditions, as shown in Ref. [26]) the latter has an asymptotic expansion as $t \downarrow 0$ :

$$
K[f](t) \sim \frac{1}{(4 \pi t)^{D / 2}} \sum_{n=0,1 / 2,1, \ldots} a_{n}[f] t^{n} .
$$

We should warn the reader that the existence of expansion (8) cannot be taken for granted. For example, in the case of some pseudodifferential operators or nonlocal boundary conditions, $\ln t$ terms appear [27,28].

If expansion (8) exists, one can take $f=1$ to define the global heat kernel coefficients:

$$
a_{n}=a_{n}[1] .
$$


If, apart from an appropriate behavior at large $t$, the heat kernel has a power-law asymptotics at small $t$, the zeta function $\zeta[f](s)$ is a meromorphic function of $s$ with simple poles. From Eqs. (7) and (8), the coefficients $a_{n}$ can be represented by the corresponding residua:

$$
a_{n}[f]={ }_{s=D / 2-n} \quad \operatorname{Res}(4 \pi)^{D / 2} \Gamma(s) \xi[f](s) \quad\left(n=0, \frac{1}{2}, 1, \ldots\right) .
$$

Furthermore, we remind the reader that, in general, the coefficients consist of a bulk (interior) and a surface (boundary) contribution

$$
a_{n}[f]=\int_{\Omega} d x f(x) b_{n}(\vec{x})+\int_{\partial \Omega} d \mu(\vec{a}) f(\vec{a}) c_{n}(\vec{a}),
$$

where we have used $\vec{a} \in \partial \Omega$ as a notation for a point on the boundary, opposite to the genuine notation $\vec{x} \in \Omega$ for a point in the bulk.

Now, we integrate Eq. (6) over the domain $\Omega$ and insert the result into Eq. (10). We thus arrive at

$$
a_{n}[f]={ }_{s=\frac{D}{2}-n}^{\text {Res }} \frac{2(4 \pi)^{D / 2}}{\Gamma(1-s)} \int_{0}^{\infty} d \omega \omega^{1-2 s} \int_{\Omega} d \vec{x} f(\vec{x}) D_{\omega}(\vec{x}, \vec{x})
$$

as the basic equation for calculating the coefficients out of the propagator.

Having briefly reviewed some basic definitions and wellknown facts, we now proceed to a brief presentation of perturbative methods.

The perturbative expansion for the resolvent is constructed in the following way: Let $D^{0}(\vec{x}, \vec{y})$ be a zeroth-order resolvent. Usually, $D^{0}$ is taken to be the free propagator in a flat space without boundaries. Consider the Dyson equation

$$
D(\vec{x}, \vec{y})=D^{0}(\vec{x}, \vec{y})+\int_{\Sigma} d \vec{z} D^{0}(\vec{x}, \vec{z}) L D(\vec{z}, \vec{y})
$$

where the integration goes over a submanifold $\Sigma \subset \Omega$, and $L$ is some operator associated with the perturbation (see examples below). Equation (13) has the formal solution

$$
\begin{aligned}
D(\vec{x}, \vec{y})= & D^{0}(\vec{x}, \vec{y})+\sum_{n=1}^{\infty} \int_{\Sigma} d \vec{z}_{1} \ldots \int_{\Sigma} d \vec{z}_{n} D^{0}\left(\vec{x}, \vec{z}_{1}\right) \\
& \times L D^{0}\left(\vec{z}_{1}, \vec{z}_{2}\right) \ldots L D^{0}\left(\vec{z}_{n}, \vec{y}\right)
\end{aligned}
$$

In Ref. [29] it was shown that the heat kernel has a similar representation,

$$
\begin{aligned}
& K(\vec{x}, \vec{y} ; t) \\
& =K_{0}(\vec{x}, \vec{y} ; t)+\sum_{n=1}^{\infty}(-1)^{n} \\
& \quad \times \int_{0}^{t} d s_{n} \int_{0}^{s_{n}} d s_{n-1} \ldots \int_{0}^{s_{2}} d s_{1} \int_{\partial M} d \mathbf{z}_{n} \ldots \int_{\partial M} d \mathbf{z}_{1} \\
& \quad \times K_{0}\left(\vec{x}, \vec{z}_{n} ; t-s_{n}\right) \\
& \quad \times L K_{0}\left(\vec{z}_{n}, \vec{z}_{n-1} ; s_{n}-s_{n-1}\right) \ldots L K_{0}\left(\vec{z}_{1}, \vec{y} ; s_{1}\right),
\end{aligned}
$$

where $K_{0}$ is a suitable chosen zeroth-order heat kernel.

In order to clarify these definitions, we consider some examples in the following discussion. Let $D^{0}=(-\mathcal{D})^{-1}$ be the propagator for a second-order differential operator $\mathcal{D}$. Let $\Sigma=\Omega$ and let $L$ be multiplied by a potential $V(\vec{x})$. This is the standard situation of the DeWitt expansion with a smooth background potential written in the form of an iterated integral equation. Then Eqs. (13)-(15) follow from the formal expansion of $D=(-\mathcal{D}+V)^{-1}$ and $K=\exp [-t(-\mathcal{D}+V)]$, respectively, so that $D(\vec{x}, \vec{y})$ and $K(\vec{x}, \vec{y} \mid t)$ are the propagator and the heat kernel of the operator $(-\mathcal{D}+V)$. If the potential $V$ is smooth and falls off sufficiently fast at infinity, all integrals in Eq. (15) exist. From dimensional considerations, it is clear that the highest power of $V$, which may contribute to the heat kernel coefficient $a_{n}$, is $V^{n}$. Therefore, only the first $n$ terms of expansion (15) must be taken into account.

In our next example, let the operator $L$ again be the multiplication by a potential $V$, but now, let $\Sigma$ be a subsurface of co-dimension 1 in $\Omega, \operatorname{dim} \Omega-\operatorname{dim} \Sigma=1 .^{1}$ In Ref. [29], it was shown that all terms in expansion (15) exist and give power-law asymptotics of the heat kernel. Later, this expansion was used in actual calculations of the heat kernel coefficients [30].

The Dyson equation is also useful for rather general perturbations of boundary conditions as, e.g., for the case where more derivative terms are added to the usual Neumann one (see [31]). In this case, however, dimensional arguments do not work, and an infinite number of terms contribute to any given heat kernel coefficient $a_{n}$.

These examples demonstrate that the "common sense" arguments work rather well. If there is a parameter $\epsilon$ in the theory such that there is a smooth limit $\epsilon \rightarrow 0$ of the heat kernel coefficients (such as $V \rightarrow 0$ above), then the formal expansions (14) and (15) in that parameter usually give a good approximation for the spectral functions. If such a parameter is of positive mass dimension, only a finite number of terms contribute to each $a_{n}$.

This is, however, not the end of the story. In Sec. III we will see that one can construct a perturbative expansion, the so-called multiple reflection expansion, even when no parameter or limiting procedure exists. Moreover, also in this case, only a finite number of terms contribute to each heat

\footnotetext{
${ }^{1}$ This problem is a particular case of a more general transmittal problem (see Sec. IV).
} 
kernel coefficient - a result that is hard to predict on the basis of common sense arguments.

\section{MULTIPLE REFLECTION EXPANSION APPLIED TO THE HEAT KERNEL COEFFICIENTS FOR THE SPHERE}

This section contains a short overview of one particular perturbative method, which is particularly well suited for the treatment of boundary problems, i.e., the multiple reflection expansion for Dirichlet and Robin boundary conditions. Balian and Bloch [22] applied this expansion, in the boundary value problem context, to calculate the density of eigenvalues, which is related to the heat kernel by a simple integral transformation. In their work [32], they pointed out that the divergent part of the Casimir energy is given by the few first reflection contributions. This fact, however, has not been fully appreciated. Therefore, we find it useful to repeat some basic facts, translating them to a more modern language, and supplying the reader with a simple example. In doing so, we omit many details that can be found in the original literature [22,33].

The multiple reflection expansion is based on simple formulas known from electrostatics: Keeping in mind the application to Dirichlet boundary conditions, let $\mu(\vec{a})$ be the density of a double layer (dipole layer) on a surface $S$. The corresponding potential is

$$
\Phi(\vec{x})=\int_{S} d^{2} a_{1} \Delta_{\omega}\left(\vec{x}-\vec{a}_{1}\right) \overleftarrow{\partial}_{a_{1}} \mu\left(\vec{a}_{1}\right),
$$

where $\overleftarrow{\partial}_{\vec{a}}$ is the normal derivative, restricted to the surface $S$ and acting to the left. Explicitly written, it reads $\Delta_{\omega}(\vec{x}$ $-\vec{a}) \overleftarrow{\partial_{a}}=\vec{n}(y) \vec{\nabla}_{y} \Delta_{\omega}(\vec{x}-\vec{y})_{\mid \vec{y}=\vec{a}}$, where $\vec{n}$ is the normal vector. The measure on $S$ is $d^{2} a_{1}=d u_{1} d u_{2} \sqrt{g}$, where $\left(u_{1}, u_{2}\right)$ are the coordinates of a point $\vec{a}\left(u_{1}, u_{2}\right)$ on $S$ and $g_{i j}$ $=\partial \vec{a} / \partial u_{i} \partial \vec{a} / \partial u_{j}$ is the metric. In Eq. (16), the propagator $\Delta_{\omega}(\vec{x}-\vec{y})$ is the free one, i.e., without boundary conditions. In three dimensions it is simply the Yukawa potential

$$
\Delta_{\omega}(\vec{x}-\vec{y})=\frac{e^{-\omega r}}{4 \pi r} \quad(r=|\vec{x}-\vec{y}|)
$$

The potential $\Phi(\vec{x})$ is discontinuous for $\vec{x}$ approaching the surface $S(\vec{x} \rightarrow \vec{a})$ and the equation

$$
\lim _{\vec{x} \rightarrow \vec{a}} \Phi(\vec{x})=\int_{S} d^{2} a_{1} \Delta_{\omega}\left(\vec{a}-\vec{a}_{1}\right) \overleftarrow{\partial}_{\vec{a}_{1}} \mu\left(\vec{a}_{1}\right)+\frac{1}{2} \mu(\alpha)
$$

holds. The additional contribution (last term) appears due to the fact that limit and integration do not commute.

In a similar fashion, keeping in mind the application to Neumann boundary conditions, the potential $\chi(\vec{x})$ of a charged surface with charge density $\rho(\vec{a})$,

$$
\chi(\vec{x})=\int_{S} d^{2} a_{1} \Delta_{\omega}\left(\vec{x}-\vec{a}_{1}\right) \rho\left(\vec{a}_{1}\right)
$$

has a discontinuous derivative:

$$
\lim _{\vec{x} \rightarrow \vec{a}} \vec{n} \vec{\nabla}_{x} \chi(\vec{x})=\int_{S} d^{2} a_{1} \vec{\partial}_{\vec{a}} \Delta_{\omega}\left(\vec{a}-\vec{a}_{1}\right) \rho\left(\vec{a}_{1}\right)-\frac{1}{2} \rho(\alpha) .
$$

In general, the multiple reflection expansion for the resolvent reads

$$
\begin{aligned}
D_{\omega}(\vec{x}, \vec{y})= & \Delta_{\omega}(\vec{x}-\vec{y}) \\
& +\kappa \int_{S} d^{2} a_{1} \Delta_{\omega}\left(\vec{x}-\vec{a}_{1}\right) \overleftrightarrow{\partial} \vec{a}_{1} \Delta_{\omega}\left(\vec{a}_{1}-\vec{y}\right) \\
& +\kappa^{2} \int_{S} d^{2} a_{1} \int_{S} d^{2} a_{2} \Delta_{\omega}\left(\vec{x}-\vec{a}_{1}\right) \\
& \overleftrightarrow{\partial}_{\vec{a}_{1}} \Delta_{\omega}\left(\vec{a}_{1}-\vec{a}_{2}\right) \overleftrightarrow{\partial}_{a_{2}} \Delta_{\omega}\left(\vec{a}_{2}-\vec{y}\right)+\ldots
\end{aligned}
$$

with the notation $\overleftrightarrow{\partial}=\overleftarrow{\partial}+\vec{\partial}$. For $\kappa=1$, this propagator obeys Dirichlet and, for $\kappa=-1$, Neumann boundary conditions. The validity of this expression can be verified by noting that it fulfills the differential equation for $\vec{x} \notin S$. Moreover, boundary conditions can be checked using Eqs. (18) and (20), whereby the additional contributions give rise to cancellations between successive orders of reflections. Expansion (21) is called multiple reflection expansion because it can be interpreted as a motion described by the free propagator from $\vec{x}$ to $\vec{a}_{1}$, being reflected (however under any angle due to the integration over $\vec{a}_{1}$ ), moving further to $\vec{a}_{2}$, and so forth. More details can be found in Ref. [33] and related papers. $^{2}$

A simple example for the multiple reflection expansion appears if the surface $S$ is a sphere. In this case, the expansion becomes an algebraic one. It can be obtained from Eq. (21) by turning to spherical coordinates. It is, however, easier to use the known expression for the exact propagator with given boundary conditions

$$
D_{\omega}\left(\vec{x}, \vec{x}^{\prime}\right)=\sum_{l, m} Y_{l, m}(\theta, \varphi) Y_{l, m}^{*}\left(\theta^{\prime}, \varphi^{\prime}\right) D_{l}\left(r, r^{\prime}\right)
$$

with

$$
D_{l}\left(r, r^{\prime}\right)=\frac{1}{\sqrt{r r^{\prime}}}\left[I_{\nu}\left(\omega r_{<}\right) K_{\nu}\left(\omega r_{>}\right)-I_{\nu}(\omega r) I_{\nu}\left(\omega r^{\prime}\right) K_{D, R}\right]
$$

and $\nu \equiv l+\frac{1}{2}, r_{<}=\min \left(r, r^{\prime}\right), r_{>}=\max \left(r, r^{\prime}\right)$. Here, $I_{\nu}(x)$ and $K_{\nu}(x)$ are the modified Bessel functions, and we have introduced the notation

\footnotetext{
${ }^{2}$ It must be stressed, that despite its simple form, the derivation of Eq. (21) contains several subtle points, which are explained in the Appendix of Ref. [33].
} 


$$
K_{D}=\frac{K_{\nu}(\omega R)}{I_{\nu}(\omega R)}
$$

for Dirichlet boundary conditions and

$$
K_{R}=\frac{(\partial / \partial R)\left[R^{u} K_{\nu}(\omega R)\right]}{(\partial / \partial R)\left[R^{u} I_{\nu}(\omega R)\right]}
$$

for Robin boundary conditions, where the solutions of Eq. (1) have to fulfill $(\partial / \partial r)\left[r^{u+1 / 2} \phi_{n}(r)\right]_{\left.\right|_{r=R}}=0$. For $u=$ $-(1 / 2)$, these reduce to Neumann boundary conditions on the two-dimensional sphere.

The multiple reflection expansion appears in the following way [33]. Represent

$$
K_{D}=\frac{K_{\nu}(\omega R) K_{\nu}^{\prime}(\omega R)}{I_{\nu}(\omega R) K_{\nu}^{\prime}(\omega R)}
$$

and use the Wronskian $I_{\nu}^{\prime}(x) K_{\nu}(x)-I_{\nu}(x) K_{\nu}^{\prime}(x)=1 / x$ to rewrite the denominator in Eq. (26) as

$$
I_{\nu}(\omega R) K_{\nu}^{\prime}(\omega R)=\frac{-1}{2 \omega R}\left(1-\omega R \frac{\partial}{\partial \omega R}\left[I_{\nu}(\omega R) K_{\nu}(\omega R)\right]\right) .
$$

Next, expand this denominator so that one obtains for $K_{D}$ the representation

$$
\begin{aligned}
K_{D}= & -2 \omega R K_{\nu}(\omega R) K_{\nu}^{\prime}(\omega R) \\
& \times \sum_{k=0}^{\infty}\left(\omega R \frac{\partial}{\partial \omega R}\left[I_{\nu}(\omega R) K_{\nu}(\omega R)\right]\right)^{k} .
\end{aligned}
$$

In a similar way, one obtains

$$
\begin{aligned}
K_{R}= & -2 \omega R K_{\nu}(\omega R)\left[K_{\nu}^{\prime}(\omega R)+\frac{u}{\omega R} K_{\nu}(\omega R)\right] \\
& \times \sum_{k=0}^{\infty}(-1)^{k}\left[\omega R \left(\frac{\partial\left[I_{\nu}(\omega R) K_{\nu}(\omega R)\right]}{\partial \omega R}\right.\right. \\
& \left.\left.+\frac{2 u}{\omega R} I_{\nu}(\omega R) K_{\nu}(\omega R)\right)\right]^{k} .
\end{aligned}
$$

This formal expansion has been shown [33] to be equivalent to the multiple reflection expansion (21), where the number of reflections is $k+1$.

In view of Eq. (12), we perform the integration over the surface of the sphere and define

$$
D_{\omega}(r)=\int_{\partial \Omega} d \mu(\vec{a}) D_{\omega}(\vec{x}, \vec{x})=\sum_{l=0}^{\infty}(2 l+1) D_{l}(r, r)
$$

so that the coefficients $a_{n}$, Eq. (9), turn out to be given by

$$
\begin{aligned}
a_{n}= & \operatorname{Res} \frac{16 \pi^{3 / 2}}{s=3 / 2-n} \int_{0}^{R} d r r^{2} \sum_{l=0}^{\infty}(2 l \\
& +1) \int_{0}^{\infty} d \omega \omega^{1-2 s} D_{l}(r, r) .
\end{aligned}
$$

The procedure to calculate the coefficients from this representation is as follows. First, we remark that the poles in $s$ result from large $\omega$ and $l$, in the Bessel functions. The poles corresponding to boundary contributions $\left[c_{n}\right.$ in Eq. (11)] appear, in addition, from the upper limit of the integration over $r$. So, we use the uniform asymptotic expansion of the Bessel functions (it is given in the Appendix), together with the multiple reflection expansion (28) or (29), and insert them into Eq. (31).

Let us start with the first contribution on the right-hand side of Eq. (23). It corresponds to the free space propagator and, thus, it does not know about the boundary. Consequently, it gives the volume contribution, which is $a_{0}$ $=(4 \pi / 3) R^{3}$.

In order to calculate the higher coefficients, we consider the second term on the right-hand side of Eq. (23). According to the sum on the right-hand side of Eqs. (28) and (29), respectively, we represent the coefficients as a sum over reflections

$$
a_{n}=\sum_{k=0}^{2 n} a_{n}^{(k)}
$$

Using the uniform asymptotic expansion of the Bessel functions, these coefficients can be calculated (for details, see Appendix A). As a result, for Dirichlet boundary conditions, the first $a_{n}^{(k)}$,s are

\begin{tabular}{llllllll}
$k=$ & 1 & 2 & 3 & 4 & 5 & 6 & $a_{n}$ \\
\hline$n=\frac{1}{2}$ & $-2 \pi^{3 / 2}$ & & & & & & $-2 \pi^{3 / 2}$ \\
$n=1$ & $2 \pi$ & $\frac{2}{3} \pi$ & & & & & $\frac{8}{3} \pi$ \\
$n=\frac{3}{2}$ & 0 & 0 & $-\frac{1}{6} \pi^{3 / 2}$ & & & $-\frac{1}{6} \pi^{3 / 2}$ \\
$n=2$ & 0 & $-\frac{4}{35} \pi$ & $-\frac{1}{21} \pi$ & $-\frac{1}{9} \pi$ & & & $-\frac{16}{315} \pi$ \\
$n=\frac{5}{2}$ & 0 & 0 & 0 & $\frac{1}{80} \pi^{3 / 2}$ & $-\frac{1}{48} \pi^{3 / 2}$ & $\frac{1}{12} \pi^{3 / 2}$ & $-\frac{1}{120} \pi^{3 / 2}$ \\
$n=3$ & 0 & $-\frac{40}{3003} \pi$ & $-\frac{2}{143} \pi$ & $\frac{12}{715} \pi$ & $-\frac{1}{130} \pi$ & $\frac{1}{90} \pi$ & $-\frac{64}{9009} \pi$
\end{tabular}


The coefficients $a_{n}$ are proportional to $R^{3-2 n}$. In this table we have taken $R=1$. When replaced in Eq. (32), they sum up to the known values (shown in the last column) as can be checked, for example, by comparing them with the results in Appendix B of Ref. [16]. It is interesting to note that the corresponding heat kernel coefficients for Robin boundary conditions with $u=0$, can be obtained through the replacement $a_{n}^{(k)} \rightarrow(-1)^{k} a_{n}^{(k)}$.

As a last example in this section, we give some reflection contributions to the heat kernel coefficient $a_{2}$ for Robin boundary conditions. They read $(R=1)$

$$
\begin{aligned}
& a_{2}^{(0)}=0, \\
& a_{2}^{(1)}=\frac{4 \pi}{105}\left(-3+12 u-28 u^{2}\right), \\
& a_{2}^{(2)}=\frac{\pi}{105}\left(5-42 u+140 u^{2}-280 u^{3}\right), \\
& a_{2}^{(3)}=\frac{\pi}{315}\left(35-270 u+756 u^{2}-840 u^{3}\right) .
\end{aligned}
$$

The sum (32) gives the known result $a_{2}=2 \pi / 45 R(1-18 u$ $\left.+60 u^{2}-120 u^{3}\right)$. In particular, Neumann boundary conditions which appear for a conducting sphere follow by choosing $u=-\frac{1}{2}$.

\section{SINGULAR POTENTIALS ON A SPHERICAL SHELL}

In this section we will study the heat kernel expansion for the free Laplacian in $\mathbb{R}^{D}$, acting on the space of functions obeying on a $D$-one-dimensional sphere, $S^{D-1}$, certain matching conditions that relate the values of the functions and their first derivatives on different sides of the sphere. If one assumes that the matching conditions are ultralocal in angular coordinates (they do not contain tangential derivatives), the most general choice is the following fourparameter family [34]:

$$
\begin{aligned}
& \phi_{+}=\omega a \phi_{-}+\omega b \phi_{-}^{\prime}, \\
& \phi_{+}^{\prime}=\omega c \phi_{-}+\omega d \phi_{-}^{\prime},
\end{aligned}
$$

where

$$
\phi_{ \pm}=\lim _{r \rightarrow R \pm 0} \phi(r), \quad \phi_{ \pm}^{\prime}=\lim _{r \rightarrow R \pm 0} \partial_{r} \phi(r) .
$$

Here, $\omega$ is a complex phase factor, which we include for completeness only. We consider real fields and put $\omega=1$. The other parameters obey the restriction $a d-b c=1$.

There are two important special cases of the conditions (33). Take

$$
a=d=1, \quad b=0 .
$$

This requires the functions to be continuous across the surface and their derivatives to have a jump. This is equivalent to having a delta function potential $V(x)=c \delta(r-R)$ on
$S^{D-1}$, which can be viewed as a singular background potential concentrated on the surface. The formal limit $c \rightarrow \infty$, turns this matching condition into Dirichlet boundary conditions $\left(\phi_{ \pm}=0\right)$.

The other special case is

$$
a=d=1, \quad c=0,
$$

requiring the derivatives to be continuous, and the functions themselves to have a jump. This is usually attributed to the presence of a background potential in the form of the derivative of the delta function. The formal limit $b \rightarrow \infty$, turns this condition into Robin boundary conditions $\left((a / b) \phi_{ \pm}+\phi_{ \pm}^{\prime}\right.$ $=0)$.

In general, the parameters $a, b, c, d$, and $\omega$ may depend on the angular coordinates on $S^{D-1}$. In this paper we restrict ourselves to the case where there is not such a dependence. Then, variables can be easily separated by making the ansatz

$$
\phi_{(n)}(x)=r^{(2-D) / 2} \phi_{n, l}(r) Y_{(l)}(\Omega),
$$

where $Y_{(l)}(\Omega)$ are the spherical harmonics depending on the angular coordinates $\Omega$. Once such ansatz is adopted, the radial functions $\phi_{n, l}$ must satisfy the equation

$$
\left[\frac{d^{2}}{d r^{2}}+\frac{1}{r} \frac{d}{d r}-\frac{\nu^{2}}{r^{2}}+\lambda_{n, l}^{2}\right] \phi_{n, l}=0
$$

with $\nu=l+(D-2) / 2$, and the matching conditions (33) at $r=R$, with shifted values of the constants:

$$
a \rightarrow \bar{a}=a+\frac{2-D}{2 R} b, \quad c \rightarrow \bar{c}=c+\frac{2-D}{2 R} d .
$$

The degeneracy of each eigenvalue $\lambda_{n, l}^{2}$ is

$$
d_{l}(D)=\frac{(2 l+D-2)(l+D-3) !}{l !(D-2) !} .
$$

In what follows, we will determine the corresponding zeta function and, from it, the corresponding heat kernel coefficients. Because we have a continuous spectrum, we must separate the translational invariant part (it does not depend on the background). We use the procedure described in Ref. [21] using the setup of a scattering off the background potential. We have to define the so-called regular solutions $\phi_{p, l}$, which have the same behavior at $r \rightarrow 0$ as the free solution

$$
\phi_{p, l}(r) \sim J_{\nu}(p r) .
$$

The behavior of this regular solution for $r \rightarrow \infty$, defines the Jost function $f_{l}(p)$ :

$$
\phi_{p, l}(r)=f_{l}(p) H^{(2)}(p r)+f_{l}^{*}(p) H^{(1)}(p r) .
$$

In the present case, the eigenfunctions of the Laplace operator can be found exactly and they give, for the problem at hand, the Jost function 


$$
\begin{aligned}
f(p)= & \frac{\pi p R}{4 i}\left[a J_{\nu}(p R) H_{\nu}^{(1)}(p R)^{\prime}+b p J_{\nu}(p R)^{\prime} H_{\nu}^{(1)}(p R)^{\prime}\right. \\
& \left.-\frac{\bar{c}}{p} J_{\nu}(p R) H_{\nu}^{(1)}(p R)-d J_{\nu}(p R)^{\prime} H_{\nu}^{(1)}(p R)\right] .
\end{aligned}
$$

Now, in order to use a formula like Eq. (4), we need to have discrete eigenvalues. So we suppose for a moment that our system is placed inside a sphere of larger radius $R^{*}$. Imposing Dirichlet boundary conditions at $r=R^{*}$, we obtain the following equation for the eigenvalues $p=\lambda_{n, l}$ :

$$
f_{l}(p) H^{(2)}\left(p R^{*}\right)+f_{l}^{*}(p) H^{(1)}\left(p R^{*}\right)=0 .
$$

Then, the $\zeta$ function can be represented as a contour integral:

$$
\begin{aligned}
\zeta(s)= & \sum_{l=0}^{\infty} d_{l}(D) \int_{\gamma} \frac{d p}{2 \pi i}\left(p^{2}+m^{2}\right)^{-s} \frac{\partial}{\partial p} \ln \left[f_{l}(p) H^{(2)}\left(p R^{*}\right)\right. \\
& \left.+f_{l}^{*}(p) H^{(1)}\left(p R^{*}\right)\right] .
\end{aligned}
$$

The contour $\gamma$ is chosen counterclockwise, enclosing all solutions of Eq. (44) on the positive real semiaxis and the positive imaginary semiaxis. For convenience, we have introduced an auxiliary mass, which we will later put to zero. There is a cut in the complex plane, which goes from im to $i \infty$. Since the number of negative modes of the Laplacian is finite, we can always choose $m$ to be sufficiently large so that all poles of the integrand (45) are below im. Next, we may deform the integration contour as described in Refs. $[16,21,18]$ to go along the two sides of the cut. We perform the limit $R^{*} \rightarrow \infty$, and drop some contributions, which are exponentially small in this limit and a term that does not depend on the matching conditions (i.e., the "empty space" contribution). The procedure sketched above is a quite general one, and not specific to this example, since it uses only some general properties of the scattering problem, such as Hermiticity and ellipticity of the Laplacian.

Next, we take the limit $m \rightarrow 0$, which is smooth at least for the heat kernel asymptotics, and obtain

$$
\zeta(s)=\frac{\sin (\pi s)}{\pi} \sum_{l=0}^{\infty} d_{l}(D) \int_{0}^{\infty} d k k^{-2 s} \partial_{k}\left[\ln f_{l}(i k)\right] .
$$

In the Jost function, we can drop any constant factor since it does not contribute to Eq. (46) and redefine

$$
f_{l}(i k)=1+\gamma k(I K)^{\prime}+\beta k^{2} I^{\prime} K^{\prime}+\alpha I K
$$

with new parameters $\gamma=(d-\bar{a}) R /(d+\bar{a}), \quad \beta=-2 b R /(d$ $+\bar{a})$, and $\alpha=2 \overline{c R} /(d+\bar{a})$, as well as the short-hand notations $I=I_{\nu}(k R)$ and $K=K_{\nu}(k R)$.

In order to get the poles of the zeta function (46), thus determining the heat kernel coefficients by means of Eq. (10), we insert into this Jost function the uniform asymptotic expansion, Eq. (A2), of the modified Bessel functions and obtain

$$
\begin{aligned}
\ln f_{l}(i k)= & \ln \left(1-\beta \frac{\nu}{2 t} i_{\nu}^{d}(t) k_{\nu}^{d}(t)+\alpha \frac{t}{2 \nu} i_{\nu}(t) k_{\nu}(t)\right. \\
& \left.+\frac{\gamma}{2}\left[i_{\nu}^{d}(t) k_{\nu}(t)-i_{\nu}(t) k_{\nu}^{d}(t)\right]\right) .
\end{aligned}
$$

Now, because all functions, $i_{\nu}(t), i_{\nu}^{d}(t), k_{\nu}(t) k_{\nu}^{d}(t)=1$ $+O(1 / \nu)$ are of order one for $\nu \rightarrow \infty$, the leading contribution in the argument of the logarithm is the one proportional to $\beta$. As this term grows with $\nu$, two cases must be treated separately, i.e., $\beta=0$ and $\beta \neq 0$.

For $\beta=0$ we obtain, by means of Eqs. (A3), an expansion similar to Eq. (A4), where the $Y_{k p i}$ are polynomials in the coefficients $\alpha$ and $\gamma$. The remaining calculations run in the same manner as in the preceding section and we obtain, in $D=3$ dimensions,

$$
\begin{aligned}
a_{1}= & -4(\alpha-\gamma) \pi, \\
a_{3 / 2}= & (\alpha-\gamma)^{2} \pi^{3 / 2}, \\
a_{2}= & -\frac{2}{15}\left(5 \alpha^{3}-120 \gamma-5 \alpha^{2} \gamma+3 \alpha \gamma^{2}-3 \gamma^{3}\right) \pi, \\
a_{5 / 2}= & \frac{1}{8}\left(\alpha^{4}-20 \alpha \gamma-2 \alpha^{3} \gamma+36 \gamma^{2}+2 \alpha^{2} \gamma^{2}\right. \\
& \left.-2 \alpha \gamma^{3}+\gamma^{4}\right) \pi^{3 / 2} .
\end{aligned}
$$

The corresponding results for higher dimensions are given in Appendix B.

Next, we turn to the case $\beta \neq 0$, which corresponds to the presence of a $\delta^{\prime}$ potential. Here, we rewrite the logarithm of the Jost function (47) in the form

$$
\begin{aligned}
\ln f_{l}(i k)= & \ln \beta+\ln \frac{\nu}{2 t}+\ln \left(1+\left(i_{\nu}^{d} k_{\nu}^{d}-1\right)-\frac{2 t}{\beta \nu}-\frac{\alpha}{\beta} \frac{t^{2}}{\nu^{2}} i_{\nu} k_{\nu}\right. \\
& \left.-\frac{\gamma}{\beta} \frac{t}{\nu}\left(i_{\nu}^{d} k_{\nu}-i_{\nu} k_{\nu}^{d}\right)\right)
\end{aligned}
$$

The first term on the right-hand side, $\ln \beta$, drops out due to the derivative in Eq. (46). The contributions surviving in the limit $\beta \rightarrow \infty$ in Eq. (50), are just the same as those one obtains for Neumann boundary conditions. Inserting now the asymptotic expressions (A3) and proceeding as above, one arrives at the following coefficients in $D=3$ dimensions:

$$
\begin{aligned}
a_{1} & =16 \frac{1}{\beta} \pi, \\
a_{3 / 2} & =\frac{1}{3}\left(1+24 \frac{\alpha}{\beta}+16 \frac{1}{\beta^{2}}\right) \pi^{3 / 2}, \\
a_{2} & =\frac{8}{15}\left(\frac{3}{\beta}+60 \frac{\alpha}{\beta^{2}}+20 \frac{\gamma}{\beta^{2}}+80 \frac{1}{\beta^{3}}\right) \pi,
\end{aligned}
$$




$$
\begin{aligned}
a_{5 / 2}= & \frac{1}{30}\left(2+40 \frac{\alpha}{\beta}+15 \frac{\gamma}{\beta}+\frac{80}{\beta^{2}}+120 \frac{\alpha^{2}}{\beta^{2}}+120 \frac{\alpha \gamma}{\beta^{2}}\right. \\
& \left.+40 \frac{\gamma^{2}}{\beta^{2}}+960 \frac{\alpha}{\beta^{3}}+480 \frac{\gamma}{\beta^{3}}+\frac{960}{\beta^{4}}\right) \pi^{3 / 2} .
\end{aligned}
$$

Again, the corresponding results for higher dimensions are given in Appendix B. As already pointed out in the Introduction, the coefficients present, in this case, a dependence on inverse powers of $\beta$.

\section{CONCLUSIONS}

In the foregoing sections we used the multiple reflection expansion as a method for the calculation of heat kernel coefficient. As a simple example, we considered boundary conditions on a sphere and obtained the heat kernel coefficients as a finite sum over reflections, Eq. (32). An interesting point is a connection between Dirichlet and Neumann boundary conditions following from this representation; the contributions from the reflections are the same in both cases except for the sign for an odd number of reflections. This can already be clearly seen from Eq. (21) and, hence, holds in general. It occurs that this seemingly simple observation has not been spelled out before.

The multiple reflection expansion, as well as the equivalent integral equations, Eq. (14) for the propagator and Eq. (15) for the heat kernel, provide a perturbative expansion. For the propagator, this expansion is convergent for imaginary frequency (as used in this paper), as was already observed in Ref. [22]. For real frequencies it may diverge. The same holds, presumably, for the heat kernel: The corresponding perturbative expansion can be expected to converge. It is interesting to note that the convergence of these expansions does not follow from a small expansion parameter. For instance, with Dirichlet boundary conditions, there is no such parameter, whereas for matching conditions corresponding to a delta function potential on the surface, there is one, cf. Ref. [29] and Sec. IV. The corresponding quantities in the expansion may be numbers that turn out to be sufficiently small. In the example with Dirichlet boundary conditions, in Eq. (28),

$$
\omega R \frac{\partial}{\partial \omega R}\left[I_{\nu}(\omega R) K_{\nu}(\omega R)\right]<1
$$

holds, ensuring the convergence of the geometric series there.

In general, the convergence issue is not trivial. As an example, we considered in Sec. IV the most general background potential concentrated on a spherical surface. It is given by the matching conditions in Eq. (33), which include a delta function potential and its derivative as special cases [Eqs. (35) and (36)]. Using the techniques introduced in Ref. [16], we calculated, for the first time, the corresponding heat kernel coefficients. The lesson with respect to the multiple reflection expansion is that, for $\beta \neq 0$ \{in Eq. (47) [or, equivalently, for $b \neq 0$ in Eq. (33)]\}, i.e., in the presence of the derivative of the delta function, the coefficients are not analytic in $\beta$. In fact, they are polynomials in $1 / \beta$. Hence, the multiple reflection expansion cannot converge for $\beta \neq 0$.

To summarize, we have stressed the convenience of using the multiple reflection expansion for the calculation of heat kernel coefficients, while showing, at the same time, some limitations of the method. In general, this method provides, after the general Seeley's calculus, the only systematic way to calculate heat kernel coefficients for manifolds with a boundary, and we expect that it will be useful in future applications.

\section{ACKNOWLEDGMENTS}

We thank K. Kirsten for helpful discussions. The work was supported by Antorchas-DAAD (Grant No. A-13740/185 ) and by the DFG (Grant No. Bo 1112/11-1).

\section{APPENDIX A}

In order to calculate the coefficients $a_{n}^{(k)}$ for $n \geqslant \frac{1}{2}$ in Eq. (32), it is useful to carry out the integration over $r$ in Eq. (31), using the known formula $\int d x x I_{\nu}(x)^{2}=x^{2} / 2\left[I_{\nu}(x)^{2}(1\right.$ $\left.\left.+\nu^{2} / x^{2}\right)-I_{\nu}^{\prime}(x)^{2}\right]$. Inserting the second term on the righthand side of Eq. (23), results in the representation

$$
\begin{aligned}
a_{n}= & \operatorname{Res} \frac{16 \pi^{3 / 2} R^{2 s}}{\Gamma(1-s)} \sum_{l=0}^{\infty} \nu^{3-2 s} \int_{0}^{\infty} d z z^{1-2 s} \\
& \times\left[I^{2}\left(1+\frac{1}{z^{2}}\right)-I^{\prime 2}\right] K_{D, R}, \\
s= & \frac{D}{2}-n
\end{aligned}
$$

where $I \equiv I_{\nu}(\nu z), K \equiv K_{\nu}(\nu z)$, and we introduced a new variable $z=\omega R / \nu$.

Next, we substitute the uniform asymptotic expansions of the modified Bessel functions for $\nu \rightarrow \infty$, $z$ fixed:

$$
\begin{aligned}
& I_{\nu}(\nu z)=\frac{1}{\sqrt{2 \pi \nu}} \frac{e^{\nu \eta}}{\left(1+z^{2}\right)^{1 / 4}} i_{\nu}(t), \\
& K_{\nu}(\nu z)=\sqrt{\frac{\pi}{2 \nu}} \frac{e^{-\nu \eta}}{\left(1+z^{2}\right)^{1 / 4}} k_{\nu}(t), \\
& I_{\nu}^{\prime}(\nu z)=\frac{e^{\nu \eta}}{\sqrt{2 \pi \nu}} \frac{\left(1+z^{2}\right)^{1 / 4}}{z} i_{\nu}^{d}(t), \\
& K_{\nu}^{\prime}(\nu z)=-\sqrt{\frac{\pi}{2 \nu}} \frac{\left(1+z^{2}\right)^{1 / 4}}{z} e^{-\nu \eta} k_{\nu}^{d}(t),
\end{aligned}
$$

with

$$
i_{\nu}(t)=\sum_{r \geqslant 0} \frac{u_{r}(t)}{\nu^{r}}, \quad k_{\nu}(t)=\sum_{r \geqslant 0} \frac{(-1)^{r} u_{r}(t)}{\nu^{r}}
$$




$$
i_{\nu}^{d}(t)=\sum_{r \geqslant 0} \frac{v_{r}(t)}{\nu^{r}}, \quad k_{\nu}^{d}(t)=\sum_{r \geqslant 0} \frac{(-1)^{r} v_{r}(t)}{\nu^{r}} .
$$

Here, the notation $t=1 / \sqrt{1+z^{2}}$ is used. The Debye polynomials $u_{r}(t)$ and $v_{r}(t)$ can be found in Ref. [35], they contain powers of $t$ from $r$ to $3 r$. We do not need the function $\eta$, since it cancels out in our case.

We can thus write

$$
\begin{aligned}
& {\left[I^{2}\left(1+\frac{1}{z^{2}}\right)-I^{\prime 2}\right] K_{D, R}} \\
& \quad=\frac{\mu^{k} 2^{-k}}{2 \nu z^{2}} \sum_{k=0}^{2 n} \sum_{p=k+1}^{2 n+1} \sum_{i=0}^{p} Y_{k p i} \frac{t^{p+2 i-1}}{\nu^{p}}+\cdots,
\end{aligned}
$$

where the coefficients $Y_{k p i}$ can be calculated easily using a simple computer program. In fact, Eq. (A4) is the definition of the $Y_{k p i}$. For Dirichlet $(\mu=+1, u=0)$ and Neumann $(\mu=-1, u=0)$ boundary conditions they are pure numbers; for Robin boundary conditions $(\mu=-1, u \neq 0)$ they are polynomials in $u$. In Eq. (A4) the dots denote higher-order terms that do not contribute to the considered heat kernel coefficients.

For the integration over $z$, we use the formula

$$
\int_{0}^{\infty} d z z^{-1-2 s} t^{-1+i}=\frac{\Gamma(-s) \Gamma\left(s+\frac{i-1}{2}\right)}{2 \Gamma\left(\frac{i-1}{2}\right)} .
$$

One can easily check that the terms with $t^{0}$ from the asymptotic expansions (A3) are canceled after substitution inside the brackets in $K_{D, R}$ [see Eqs. (28) and (29)]. This means that any new reflection contributes at least one power of $t$ to the integrand in Eq. (A1). Therefore, according to Eq. (A5), only several first terms of the multiple reflection expansion contribute to any given heat kernel coefficient. This explains the finite range of the summations in Eq. (A4).

The sum over $l$ produces Hurwitz zeta functions. When taking this into account, we obtain for the contribution of $k$ reflections to $a_{n}$ [see Eq. (32)]:

$$
\begin{aligned}
a_{n}^{(k)}= & \operatorname{Res} \frac{16 \pi^{3 / 2}}{\Gamma(1-s)} \sum_{p=k+1}^{2 n+1} \sum_{i=0}^{p} Y_{k p i} \frac{R^{2 s} \mu^{k+1}}{2^{k+1}} \\
& \times \zeta\left(2 s+p-2, \frac{1}{2}\right) \frac{\Gamma(-s) \Gamma\left(s+i+\frac{p-1}{2}\right)}{2 \Gamma\left(i+\frac{p-1}{2}\right)}, \\
s= & \frac{3}{2}-n .
\end{aligned}
$$

The calculation of the residua can be carried out, again, using standard computer algebra programs, which lead to the coefficients $a_{n}^{(k)}$ in Eq. (32).

\section{APPENDIX B}

Here we present the results for the heat kernel coefficients corresponding to the matching conditions in Sec. IV, in the cases of some higher-dimensional spaces. For $\beta=0$, we obtain, instead of Eq. (49), in $D=4$ dimensions,

$$
\begin{aligned}
a_{1}= & -2(\alpha-\gamma) \pi^{2}, \\
a_{3 / 2}= & \frac{1}{2}(\alpha-\gamma)^{2} \pi^{\frac{5}{2}}, \\
a_{2}= & -\frac{1}{6}\left(2 \alpha^{3}-105 \gamma-2 \gamma^{3}\right) \pi^{2}, \\
a_{5 / 2}= & \frac{1}{64}\left(-3 \alpha^{2}+4 \alpha^{4}-172 \alpha \gamma-4 \alpha^{3} \gamma+298 \gamma^{2}\right. \\
& \left.+3 \alpha^{2} \gamma^{2}-10 \alpha \gamma^{3}+7 \gamma^{4}\right) \pi^{5 / 2}
\end{aligned}
$$

in $D=5$ dimensions,

$$
\begin{aligned}
& a_{1}=\frac{-8}{3}(\alpha-\gamma) \pi^{2}, \\
& a_{3 / 2}=\frac{2}{3}(\alpha-\gamma)^{2} \pi^{5 / 2}, \\
& a_{2}=-\frac{4}{45}\left(5 \alpha^{3}-465 \gamma+5 \alpha^{2} \gamma-\alpha \gamma^{2}-9 \gamma^{3}\right) \pi^{2}, \\
& a_{5 / 2}=\frac{1}{12}\left(-2 \alpha^{2}+\alpha^{4}-76 \alpha \gamma+128 \gamma^{2}-4 \alpha \gamma^{3}+3 \gamma^{4}\right) \pi^{5 / 2}
\end{aligned}
$$

in $D=6$ dimensions,

$$
\begin{aligned}
a_{1}= & -(\alpha-\gamma) \pi^{3}, \\
a_{3 / 2}= & \frac{1}{4}(\alpha-\gamma)^{2} \pi^{7 / 2}, \\
a_{2}= & -\frac{1}{12}\left(6 \alpha+2 \alpha^{3}-297 \gamma+4 \alpha^{2} \gamma-6 \gamma^{3}\right) \pi^{3}, \\
a_{5 / 2}= & \frac{1}{128}\left(\alpha^{2}+4 \alpha^{4}-508 \alpha \gamma+4 \alpha^{3} \gamma+802 \gamma^{2}-\alpha^{2} \gamma^{2}\right. \\
& \left.-26 \alpha \gamma^{3}+19 \gamma^{4}\right) \pi^{7 / 2} ;
\end{aligned}
$$

and in $D=7$ dimensions,

$$
a_{1}=-\frac{16}{15}(\alpha-\gamma) \pi^{3}
$$




$$
\begin{aligned}
a_{3 / 2}= & \frac{4}{15}(\alpha-\gamma)^{2} \pi^{7 / 2}, \\
a_{2}= & -\frac{8}{225}\left(5 \alpha^{3}-1050 \gamma+15 \alpha^{2} \gamma+3 \alpha \gamma^{2}-23 \gamma^{3}\right) \pi^{3}, \\
a_{5 / 2}= & \frac{1}{30}\left(-6 \alpha^{2}+\alpha^{4}-172 \alpha \gamma+2 \alpha^{3} \gamma+280 \gamma^{2}-10 \alpha \gamma^{3}\right. \\
& \left.+7 \gamma^{4}\right) \pi^{7 / 2} .
\end{aligned}
$$

For $\beta \neq 0$ we obtain, instead of Eq. (51), in $D=4$ dimensions,

$$
\begin{aligned}
a_{1}= & \frac{8}{\beta} \pi^{2}, \\
a_{3 / 2}= & \frac{1}{16}\left(9+64 \frac{\alpha}{\beta}-32 \frac{\gamma}{\beta}+\frac{128}{\beta^{2}}\right) \pi^{5 / 2}, \\
a_{2}= & \frac{16}{3}\left(3 \frac{\alpha}{\beta^{2}}+\frac{4}{\beta^{3}}\right) \pi^{2}, \\
a_{5 / 2}= & \frac{1}{2048}\left(-59-512 \frac{\alpha}{\beta}-224 \frac{\gamma}{\beta}-512 \frac{1}{\beta^{2}}+4096 \frac{\alpha^{2}}{\beta^{2}}\right. \\
& +2048 \frac{\alpha \gamma}{\beta^{2}}+512 \frac{\gamma^{2}}{\beta^{2}}+32768 \frac{\alpha}{\beta^{3}}+8192 \frac{\gamma}{\beta^{3}} \\
& \left.+32768 \frac{1}{\beta^{4}}\right) \pi^{5 / 2} ;
\end{aligned}
$$

in $D=5$ dimensions,

$$
\begin{aligned}
a_{1} & =\frac{32}{3} \frac{\pi^{2}}{\beta}, \\
a_{3 / 2} & =\frac{2}{3}\left(3+2 \frac{\alpha}{\beta}-2 \frac{\gamma}{\beta}+\frac{4}{\beta^{2}}\right) \pi^{5 / 2}, \\
a_{2} & =\frac{16}{45}\left(-\frac{1}{\beta}+60 \frac{\alpha}{\beta^{2}}-20 \frac{\gamma}{\beta^{2}}+\frac{80}{\beta^{3}}\right) \pi^{2},
\end{aligned}
$$

$$
a_{5 / 2}=\frac{4}{3}\left(-\frac{17}{240}-\frac{\alpha}{\beta}-\frac{2}{\beta^{2}}+2 \frac{\alpha^{2}}{\beta^{2}}+16 \frac{\alpha}{\beta^{3}}+\frac{16}{\beta^{4}}\right) \pi^{5 / 2} ;
$$

in $D=6$ dimensions,

$$
\begin{aligned}
a_{1}= & \frac{4}{\beta} \pi^{3}, \\
a_{3 / 2}= & \frac{1}{32}\left(\frac{191}{3}+64 \frac{\alpha}{\beta}-96 \frac{\gamma}{\beta}+\frac{128}{\beta^{2}}\right) \pi^{7 / 2}, \\
a_{2}= & \frac{2}{3}\left(\frac{3}{\beta}+12 \frac{\alpha}{\beta^{2}}-8 \frac{\gamma}{\beta^{2}}+\frac{16}{\beta^{3}}\right) \pi^{3}, \\
a_{5 / 2}= & \frac{1}{12288}\left(103+512 \frac{\alpha}{\beta}-480 \frac{\gamma}{\beta}+\frac{512}{\beta^{2}}+12288 \frac{\alpha^{2}}{\beta^{2}}\right. \\
& -6144 \frac{\alpha \gamma}{\beta^{2}}-512 \frac{\gamma^{2}}{\beta^{2}}+98304 \frac{\alpha}{\beta^{3}}-24576 \frac{\gamma}{\beta^{3}} \\
& \left.+\frac{98304}{\beta^{4}}\right) \pi^{7 / 2} ;
\end{aligned}
$$

and in $D=7$ dimensions,

$$
\begin{aligned}
a_{1}= & \frac{64}{15} \frac{\pi^{3}}{\beta}, \\
a_{3 / 2}= & 4\left(10+8 \frac{\alpha}{\beta}-16 \frac{\gamma}{\beta}+\frac{16}{\beta^{2}}\right) \pi^{7 / 2} \\
a_{2}= & \frac{32}{225}\left(3+60 \frac{\alpha}{\beta^{2}}-60 \frac{\gamma}{\beta^{2}}+\frac{80}{\beta^{3}}\right) \pi^{3}, \\
a_{5 / 2}= & \frac{4}{15}\left(-\frac{15}{8}-6 \frac{\alpha}{\beta}+6 \frac{\gamma}{\beta}-\frac{12}{\beta^{2}}+4 \frac{\alpha^{2}}{\beta^{2}}\right. \\
& \left.-4 \frac{\alpha \gamma}{\beta^{2}}+32 \frac{\alpha}{\beta^{3}}-16 \frac{\gamma}{\beta^{3}}+\frac{32}{\beta^{4}}\right) \pi^{7 / 2} .
\end{aligned}
$$

[1] I. Avramidi, Heat Kernel and Quantum Gravity, Springer Lecture Notes in Physics m64 (Springer, Berlin, 2000).

[2] V. A. Fock, Izvestiya of USSR Academy of Sciences (Phys.) 4-5, 551 (1937).

[3] J. Schwinger, Selected Papers on Quantum Electrodynamics (Dover, New York, 1958).

[4] B. S. DeWitt, Dynamical Theory of Groups and Fields (Gordon and Breach, New York, 1965).

[5] P. Amsterdamski, A. L. Berkin, and D. J. O'Connor, Class. Quantum Grav. 6, 1981 (1989).
[6] I. G. Avramidi, Phys. Lett. B 238, 92 (1990).

[7] I. G. Avramidi, Nucl. Phys. B355, 712 (1991); B509: 577(E) (1998).

[8] A. E. M. van de Ven, Class. Quantum Grav. 15, 2311 (1998).

[9] P. B. Gilkey, J. Diff. Geom. 10, 601 (1975).

[10] T. P. Branson and P. B. Gilkey, Commun. Partial Diff. Eqns. 15, 245 (1990).

[11] P. B. Gilkey, Invariance Theory, the Heat Equation, and the Atiya-Singer Index Theorem (CRC, Boca Raton, FL, 1995).

[12] T. P. Branson, P. B. Gilkey, K. Kirsten, and D. V. Vassilevich, 
Nucl. Phys. B563, 603 (1999).

[13] P. B. Gilkey, K. Kirsten, and D. V. Vassilevich, Nucl. Phys. B601, 125 (2001).

[14] V. D. Lyakhovsky, N. N. Shtykov, and D. V. Vassilevich, Lett. Math. Phys. 21, 89 (1991).

[15] D. V. Vassilevich and N. N. Shtykov, Yad. Fiz. 53, 869 (1991) [Sov. J. Nucl. Phys. 53, 542 (1991)].

[16] M. Bordag, E. Elizalde, and K. Kirsten, J. Math. Phys. 37, 895 (1996).

[17] G. Esposito, A. Yu. Kamenshchik, and G. Pollifrone, Euclidean Quantum Gravity on Manifolds with Boundary (Kluwer, Dordrecht, 1997).

[18] Klaus Kirsten, "Spectral functions in mathematics and physics," hep-th/0007251.

[19] I. G. Avramidi and G. Esposito, Class. Quantum Grav. 15, 281 (1998).

[20] J. S. Dowker and K. Kirsten, Class. Quantum Grav. 16, 1917 (1999).

[21] M. Bordag and K. Kirsten, Phys. Rev. D 53, 5753 (1996).

[22] R. Balian and C. Bloch, Ann. Phys. (N.Y.) 60, 401 (1970); 84, 559(E) (1974).
[23] D. M. McAvity and H. Osborn, Class. Quantum Grav. 8, 603 (1991).

[24] O. D. Kellogg, Foundations of Potential Theory (SpringerVerlag, Berlin, 1929).

[25] C. Grosche, J. Phys. A 28, L99 (1995).

[26] P. Greiner, Arch. Ration. Mech. Anal. 41, 163 (1971).

[27] G. Grubb and R. Seeley, Invent. Math 121, 481 (1995).

[28] P. B. Gilkey and G. Grubb, Commun. Partial Diff. Eqns. 23, 777 (1998).

[29] M. Bordag and D. V. Vassilevich, J. Phys. A 32, 8247 (1999).

[30] Ian G. Moss, Phys. Lett. B 491, 203 (2000).

[31] W. Kummer and D. V. Vassilevich, J. High Energy Phys. 07, $012(2000)$.

[32] R. Balian and B. Duplantier, Ann. Phys. (N.Y.) 112, 165 (1978).

[33] T. H. Hansson and R. L. Jaffe, Ann. Phys. (N.Y.) 151, 204 (1983).

[34] S. Albeverio, F. Gesztesy, R. Høegh-Krohn, and H. Holen, Solvable Models in Quantum Mechanics (Springer-Verlag, New York, 1988).

[35] M. Abramowitz and I. A. Stegun, Handbook of Mathematical Functions (Dover, New York, 1970). 